\title{
La competencia internacional y el cambio de la matriz de producción ${ }^{1}$
}

\section{International competition and the change of the production matrix}

\author{
Pedro Barrientos Felipa ${ }^{1}$
}

\begin{abstract}
RESUMEN
La proyección comercial exportadora de un país implica reconocer la importancia del comercio así como de la competencia o otros países proveedores que buscan abastecer al mismo mercado objetivo. Para competir un país debe diversificar su matriz de producción motivo por el cual debe aumentar su producción y añadir nuevas líneas de negocios. Un sector que tiene expectativas en Perú es la agricultura la que está diversificándose por las expectativas del inversionistas. La propuesta es que el Estado debe actuar de manera coordinada con todos los sectores como un agente que permita conocer cuánto de apoyo se necesita para estar en capacidad de competir y los recursos que son necesarios en el tiempo. La participación debe favorecer a todos los agentes que participan directamente, lo que incluye al Estado
\end{abstract}

1 Este artículo de revisión es resultado del proyecto de investigación titulado "Los productos agrarios en la estrategia del Plan Nacional de Diversificación Productiva " realizada en el año 2016. La investigación fue auspiciada por el Instituto de Investigaciones Económicas de la Facultad de Ciencias Económicas contando con el financiamiento de la Universidad Nacional Mayor de San Marcos.

2 Economista, Universidad Ricardo Palma, Lima, Perú. Magíster con Concentración en Marketing, Universidad del Pacífico, Lima, Perú. Doctor en Administración de Negocios Globales, Universidad Ricardo Palma, Lima, Perú. Vice Decano de la Unidad de Investigación y Posgrado, Profesor principal y Miembro del Instituto de Investigaciones Económicas, Facultad de Ciencias Económicas, Universidad Nacional Mayor de San Marcos, Lima, Perú. Dirección postal: Calle Germán Amézaga 375, Edificio Facultad de Ciencias Económicas. Teléfono 051-998648480. Correo electrónico: pbarrientosf@ unmsm.edu.pe.

(C) Los autores. Este artículo es publicado por Pensamiento Crítico de la Facultad de Ciencias Económicas, Universidad Nacional Mayor de San Marcos. Este es un artículo de acceso abierto, distribuido bajo los términos de la licencia Creative Commons Atribucion - No Comercia_Compartir Igual 4.0 Internacional. (http:// creativecommons.org/licenses/by-nc-sa/4.0/) que permite el uso no comercial, distribución y reproducción en cualquier medio, siempre que la obra original sea debidamente citada. 
y los pequeños agricultores. La competencia, la diversificación y la importancia de los agentes productivos es lo que busca exponer el artículo.

Palabras clave: Competencia internacional; marca país; diversificación productiva; pequeño agricultor

Clasificación: JELM19, M31

\section{ABSTRACT}

The exporting commercial projection of a country implies recognizing the importance of trade as well as competition or other supplier countries that seek to supply the same target market. To compete a country must diversify its production matrix, which is why it must increase its production and add new lines of business. A sector that has expectations in Peru is agriculture that is diversifying by the expectations of investors. The proposal is that the State must act in a coordinated manner with all sectors as an agent that allows knowing how much support is needed to be able to compete and the resources that are necessary over time. Participation should favor all agents that participate directly, including the State and small farmers. The competition, the diversification and the importance of the productive agents is what the article seeks to expose.

Key words: International competition; country brand; productive diversification; small farmer

\section{Introducción}

Al igual que las empresas buscan posicionarse como ellas mismas o sus productos en los compradores extranjeros, los países también buscan realizar la misma acción con la finalidad de mejorar su imagen como país y sus ventas al exterior. La evidencia explica que hoy hay más países por querer participar en el comercio internacional y los gobiernos buscando que esta decisión se convierta en una acción permanente y sostenible. Entienden que para que la estrategia funcione deben considerar establecerse como una marca, al igual que lo hacen las empresas y sus productos. Kotler y Armstrong (2013, p. 196) definen un producto "como algo que puede ser ofrecido a un mercado para su atención, adquisición, uso o consumo, y que podría satisfacer un deseo o una necesidad". Administrar un país y establecerlo como una marca es un proceso más complejo que la administración de una marca producto. Desarrollar el marketing de un país a través de sus actividades económicas debe llevar a lograr una marca país. 
Para una empresa desarrollar estrategias de marketing para sus productos es un proceso complejo debido a que tiene que conjugar diversos intereses internos (producción, logística, marketing, recursos humanos, finanzas) y externos (distribuidores, proveedores, prestadores de servicios logísticos, servicios financieros) de modo que asegure el futuro del producto de manera sostenible y así se convierta en un producto de marca o un producto posicionado. Los países pueden optar también por establecerse como un producto de marca o un país de marca y el camino que sigue es el mismo que se cita para las empresas, al que se denomina actividades de marca país. El camino implica decisiones de política de Estado que los gobiernos sucesivos deben continuamente mejorar. Si en un producto el producto el posicionamiento puede demorar años, en un país el proceso demora décadas. Por tanto, sus actividades, como país, requieren del diseño de múltiples estrategias para lograrlo y debe ser consensuado entre los diversos agentes que influyen en él.

En el mundo globalizado la competencia comercial entre los países se intensifica, en especial en los países latinoamericanos. Los países consideran pertinente no sólo actuar como productores primarios, sino que promueven añadir nuevas líneas a su sistema de producción considerando las necesidades de los consumidores de los países compradores, motivo por el cual se optan por un proceso de modificación de la matriz de producción, sin abandonar la exportación de productos primarios. La idea de modificación de matriz productiva, con la finalidad de aprovechar la posibilidad de proveer a mercados extranjeros, es parte de la política económica de varios países y la consecuencia es la intensificación de la competencia internacional, es decir, varios productores compiten por ser el proveedor de un mercado objetivo.

Un país se asegura el liderazgo en un mercado específico si se establece como marca, si logra distinguirse no sólo en el producto sino en toda su cadena de valor, es decir desde la provisión hasta la distribución. Barrientos menciona (2014, p. 118) que "establecer una marca país como parte de una estrategia de marketing país es un proceso de creación de una oferta de productos tangibles más intangibles. Implica disputar competitivamente. Hay varios proveedores para la satisfacción de la demanda, actual y potencial". Para asegurar la compra los países realizan actividades de diversas actividades macro para lograr un posicionamiento. 
Lo que permite que la producción de un país se considere como sinónimo de "buen producto" o el reconocimiento internacional de lo que se hace en el país "es "sinónimo de calidad". El proceso es complejo, de diseñar el largo como país y se puede observar el conjunto de actividades que se tienen que realizar en el siguiente gráfico:

SOPORTE DE LAS ACTIVIDADES DE DIFERENCIACIÓN:

LO QUE DEBE HACER UN PAÍS

Sistema de educación, justicia legal, ocupación, tecnología, investigación, alimentación, desarrollo infantil, sistema de salud, protección a la herencia cultural, estabilidad política, seguridad interna, matriz de producción, desconcentración y descentralización geográfica y política.

ACTIVIDADES DE DIFERENCIACIÓN
Mezcla de marketing país
Personas o los ciudadanos del país; los
productos o alternativas de consumo para los
mercados objetivo; promoción o comunicación;
los procesos o el cómo se hacen las cosas; las
relaciones internacionales; infraestructura física;
conservación de los lugares; las organizaciones
del Estado y del sector privado.

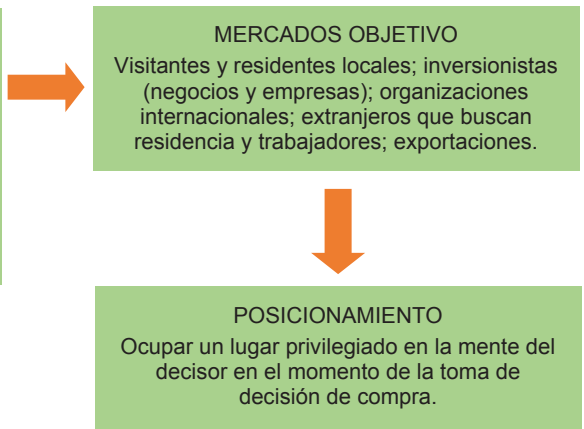

Figura 1. Construcción del posicionamiento de un país Fuente: Barrientos (2014, p. 164)

Una de las actividades que se realizan para la construcción del posicionamiento es lo que corresponde a las modificaciones de la matriz de producción de productos para el consumo local y del extranjero. El país necesita dar mayor énfasis a otros productos que le permiten diferenciarse de concepto de primario exportador. Debe ingresar a un proceso de diversificación añadiendo nuevas líneas o actividades productivas. Por ejemplo, que a Perú se le reconozca además de exportador de minerales como un proveedor de otros productos, que expongan a los compradores internacionales que no sólo venden productos básicos. Hausmann e Hidalgo (2010, p. 2) comparten la propuesta cuando mencionan que "The 
more capabilities a country has, the higher the return, in terms of increased diversification, that the accumulation of a new capability will provide given that the possible combination of any additional capability with existing ones grows exponentially with the number of capabilities already available in a country". Por tanto, la diversificación productiva es una buena elección por que añaden nuevas actividades económicas y por su efecto multiplicador, es decir fortalece la cadena productiva del país.

Sin embargo, hay dos decisiones claves que tienen que tomarse para que la diversificación apoye reformular el posicionamiento del país. La primera es si el proceso de la diversificación es consensuado en cuanto a la identificación de las actividades necesarias, que involucran compromisos de los elementos integrantes de la cadena de valor del país. La segunda corresponde a las actividades económicas que tienen ventajas comparativas que pueden convertirse en ventajas competitivas, lo cual no significa que dejan de tener importancia para la economía, sino que se convierten en el respaldo a la decisión tomada.
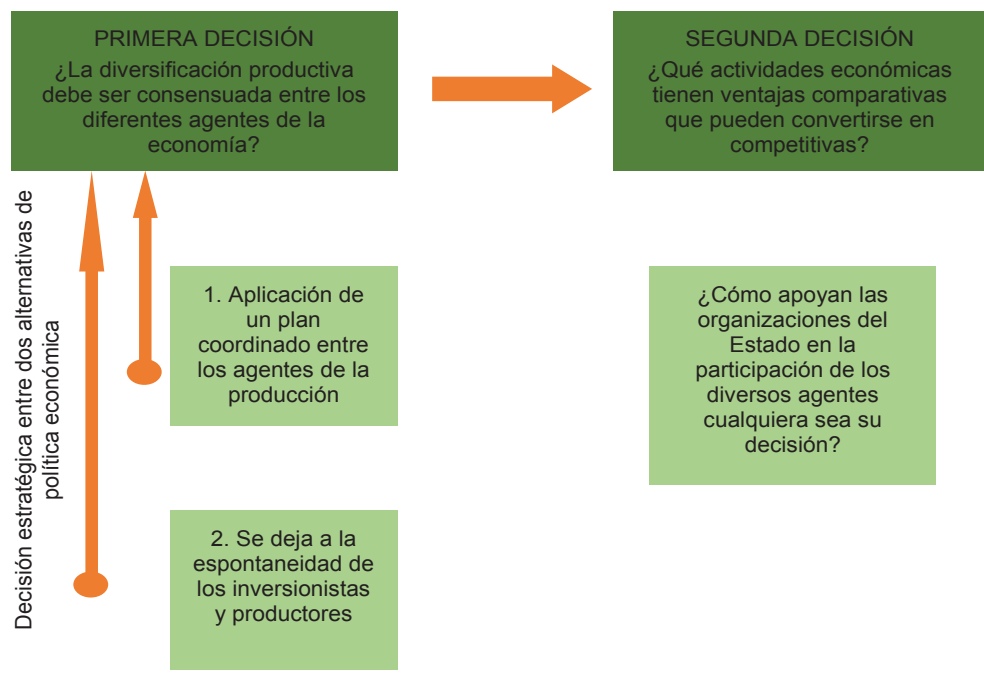

Elaboración: propia

Figura 2. Las decisiones para diversificar las actividades económicas Fuente: Barrientos (2014, p. 164) 
En el caso de la primera decisión, queda en elegir, por parte de los agentes económicos involucrados, si la diversificación productiva es una actividad planificada en la que participan todos los agentes involucrados de la economía local como el Estado, los inversionistas privados, los productores (grandes, medianos y pequeños), instituciones financieras, organizaciones sociales, que tienen un objetivo común acerca del país frente a los competidores que también buscan obtener mercados, conseguir y mantener clientes, tener una consolidada imagen entre sus compradores actuales y potenciales, así como reconocimiento internacional. La otra alternativa es dejar a la espontaneidad del inversionista privado quien decide a que sector va de acuerdo al mercado objetivo que identifica para realizar sus ventas.

La otra decisión, corresponde a un aspecto macroeconómico, en cuanto cuáles son los sectores o actividades económicas en que se tiene ventajas comparativas y luego convertirlas en competitivas. Al respecto el Ministerio de la Producción (2014) en su proyecto Plan Nacional de Diversificación Productiva (PNDP) expone las diversas actividades económicas con un potencial para el comercio exterior (Cuadro 1), destacando la actividad agrícola, la que se identifica por estar en cuatro de las cinco regiones en las que se realizó el estudio respectivo. Como se describe en el Cuadro 1, la actividad agrícola, no procesada y procesada, tiene un potencial que puede ser aprovechado para el desarrollo del comercio internacional.

\section{El concepto de competir y diversificación productiva}

Competir a nivel macro en el campo internacional implica poner en práctica una política de Estado que implica modificar la conducta de producción interna orientada hacia satisfacer necesidades del comprador y del consumidor en el exterior. Es un cambio relativo, que es sostenible en el tiempo, de la matriz de producción considerando a los compradores para su diseño. Es seguir produciendo los productos primarios pero impulsar otros productos que también tienen posibilidades en el comercio internacional. En el caso de los productos no primarios como la agricultura implica incrementar la producción, la productividad, la eficiencia y otorgar un mayor valor agregado a sus productos. Esta transformación toma tiempo en mostrar los resultados. 
Pensamiento Crítico Vol. 22. $\mathrm{N}^{\circ} 2$

Cuadro 1

Identificación de oportunidades en el nivel de regiones

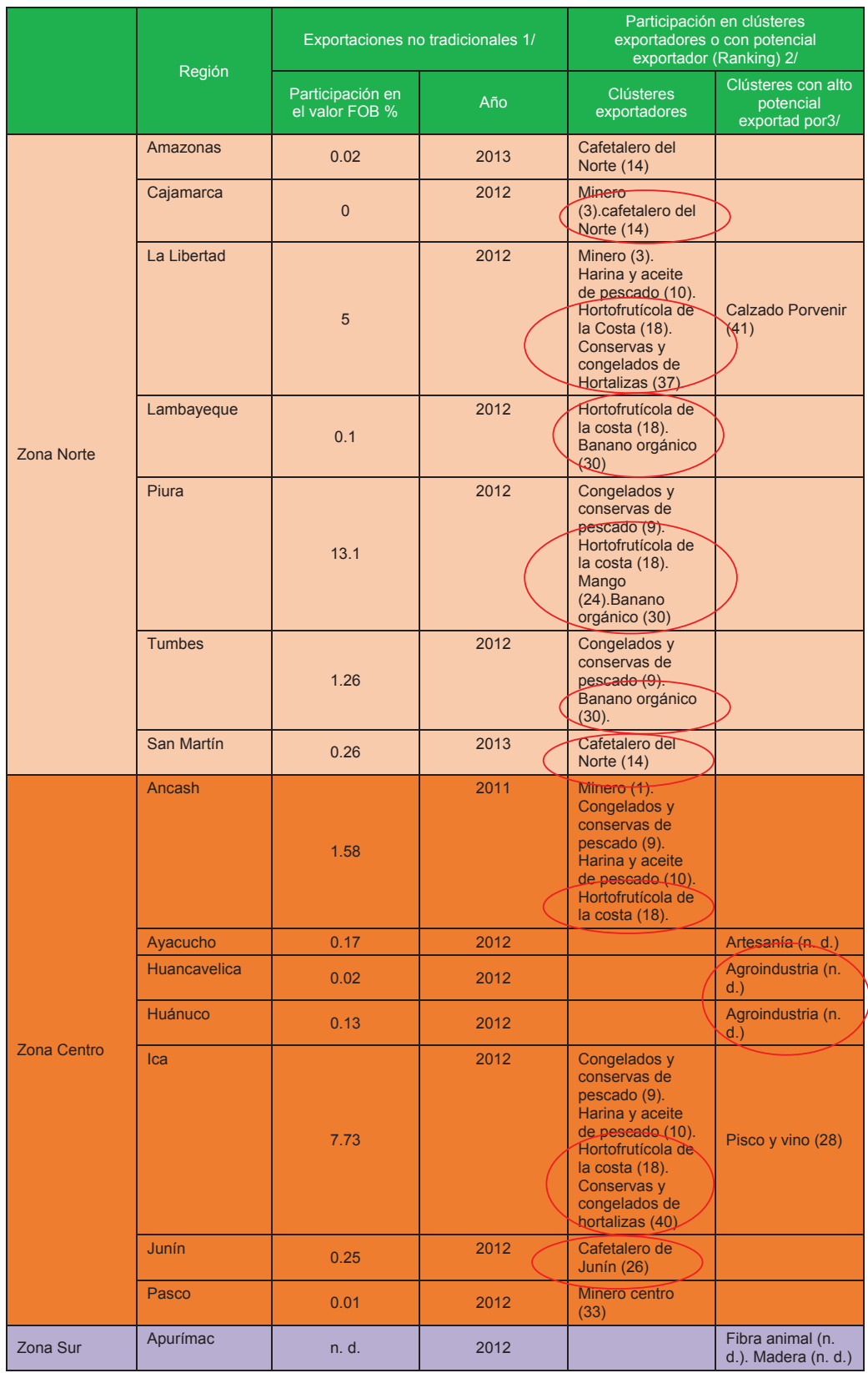




\section{PEDRo BARRIENTos Felipa}

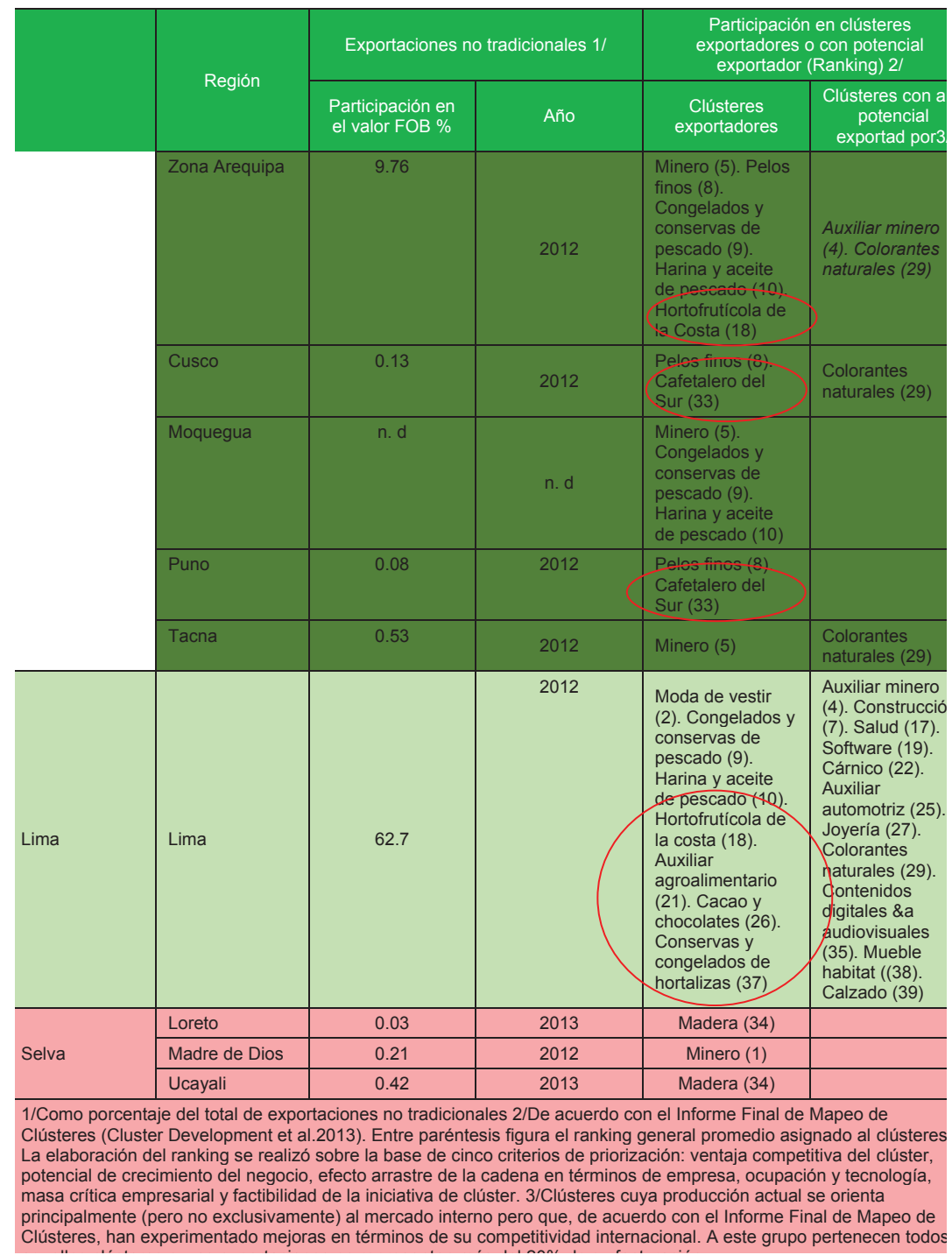

Fuente: Ministerio de la Producción (2014, p. 71).

Nota: Los círculos en el Cuadro 1 son añadidos por el autor del artículo 
Alarco (2015), respecto a la propuesta del Ministerio de la Producción (2014), menciona que la estrategia tal como está planteada tiene sus limitaciones y deben ser superadas. Cita como paso inicial la convocatoria de expertos de todos los sectores y de los representantes empresariales que analicen situaciones transversales. También que es conveniente el reconocimiento del papel de la ciencia y la tecnología, de la innovación y de la capacitación especializada. Se debe sumar el establecimiento de criterios para priorizar determinadas actividades y la priorización de los recursos limitados.

Lo que corresponde plantearse luego de la modificación de la matriz productiva en la agricultura es la posibilidad de dar valor agregado a estos productos, de acuerdo a sus características. Algunos de los productos que se exportan la transformación o la realización de valor agregado se realiza en el punto de destino, en el lugar de residencia de los compradores. Otros productos no tienen ningún añadido. Como país, llega el momento de decidir a qué producto se le otorga valor agregado y a quienes se venden tal como está. Si al otorgarse valor agregado a un producto en su punto de origen el margen de ganancia aumenta es factible realizarlo, caso contrario se deja de lado.

Machado (2014) hace hincapié en la importancia de los recursos financieros en cantidad suficiente que permita que se cumpla lo planificado. El Plan Nacional de Diversificación Productiva (PNDP) menciona que Perú tiene una diversidad de factores que tienen que ser fortalecidos si se considera necesario contar con una estrategia sostenible como un motor de crecimiento. Las actividades que son necesarias tienen un costo monetario. Si la iniciativa de la diversificación se deja al inversionista, éste tiene un comportamiento atomizado motivo por el cual se logra una diversificación más lenta, mientras que si la acción es planificada a través del sector privado (inversionistas y productores) y el Estado la posibilidad de hacerlo más rápido mejora.

Competir en el comercio internacional implica que el país ha definido las acciones necesarias para lograr un objetivo consolidado en la internacionalización de sus productos. El resultado es que aumenta la rentabilidad de los inversionistas y productores, y genera recursos al Estado para sus desarrollar las acciones sociales que le corresponden, 
siendo resultado la reformulación de su posicionamiento como país. Para tal caso, el PNDP propone interrelacionar un conjunto de instituciones públicas y privadas con la finalidad de lograr objetivos consensuados. Esto ocurre para cada uno de los sectores de la economía peruana.

En la agricultura peruana, se ha realizado un proceso de diversificación llevada a cabo por inversionistas privados como un cambio en la matriz de producción agraria. Gómez (2016, p. 30) cita que en la década de los años del siglo XX el espárrago representaba el $30 \%$ de la agroexportación no tradicional. A inicios de este siglo, en su primera década tienen un crecimiento significativo la producción de uvas, las paltas y los mangos. Sin embargo, hay otros productos agrícolas que tiene un fuerte impulso para ofertar al mercado internacional como el banano, alcachofa, cacao, paprika, quinua, entre otros. En tal sentido, la diversificación en la producción agraria busca que se incremente la cartera de productos agrícolas y a la vez aumente la producción exportable, siendo la consecuencia de un mayor relativo en la matriz de producción nacional.

La suma de las estrategias y su operatividad en el comercio internacional determinan el aprovechamiento de las oportunidades de mercado. La diversificación en el sector agricultura implica también que también los otros sectores, que son parte de la cadena de valor, también se diversifiquen. El PNDP y su aceptación implican un cambio estructural de la economía peruana, en donde participan los grupos de interés de manera mancomunada, lo que lo hace un proceso complejo. El Estado, a través del gobierno central, tiene que comprometerse a su cumplimiento, considerando la propuesta de Huasmann y Rodrik (2002, p. 35):

This is a task at which many governments have proved inadequate. But in the absence of a policy framework that is cognizant of this obstacle, adoption of good institutions and openness to foreign technology may well prove insufficient to spark a sustained process of economic transformation and growth.

El proceso complejo de añadir una nueva forma de producir para exportar para competir, adoptando como estrategia la diversificación expone la diversidad de organismos públicos que participan para el cumplimiento de los objetivos que se plantean. Existe una diversidad de asignación de recursos institucionales, personas, procesos y financieros, siendo una exposición del proceso complejo y su administración lo que expone por Alarco (2012, p.245): 
En el campo de lo más instrumental, la implantación de políticas industriales en el Perú requiere un marco institucional apropiado que comprenda una institución al máximo nivel de gobierno y de un marco normativo que haga viables estas políticas a favor de la diversificación productiva. Por otra parte, se requiere de personal idóneo y calificado para desarrollar y conducir esta política, así como la existencia de empresas de asesoría y consultoría privadas que se encargarían de realizar el trabajo directamente con las empresas con potencial que habría que impulsar. Por último, habría que disponer de los recursos económicos y financieros para diseñar e implantar las políticas específicas.

\section{La matriz agrícola peruana y las ventas en el mercado internacional}

En el año 2005, tal como se observa en el Cuadro 2 las exportaciones agrícolas tradicionales representan el 1,9\% del total exportado; en el año 2015 representan el 2,1\%. Las exportaciones no tradicionales en el 2005 representaban el 5,8 \% del monto exportable, en el 2015 representan el $12,8 \%$. La explicación de los cambios en la matriz agrícola exportable se explica por la presencia en nuevos cultivos que a su vez son requeridos por los compradores internacionales lo que incentiva a la producción de nuevos productos agrícolas. Sin embargo, este resultado no logra disminuir el riesgo relativo que implican las exportaciones mineras.

\section{Cuadro 2}

Participación de los productos agrícolas en la matriz de exportación

\begin{tabular}{|l|c|c|c|c|c|c|c|c|c|c|c|}
\hline Años & 2005 & 2006 & 2007 & 2008 & 2009 & 2010 & 2011 & 2012 & 2013 & 2014 & 2015 \\
\hline $\begin{array}{l}\text { EXPORTACIONES } \\
\begin{array}{l}\text { Valores FOB en } \\
\text { millones US\$ }\end{array}\end{array}$ & $\mathbf{1 7 3 6 8}$ & $\mathbf{2 3} \mathbf{8 3 0}$ & $\mathbf{2 8 ~ 0 9 4}$ & $\mathbf{3 1 ~ 0 1 8}$ & $\mathbf{2 6} \mathbf{9 6 2}$ & $\mathbf{3 5} \mathbf{5 6 5}$ & $\mathbf{4 6} \mathbf{2 6 8}$ & $\mathbf{4 5} \mathbf{6 3 9}$ & $\mathbf{4 2 . 8 6 1}$ & $\mathbf{3 9 . 5 3 3}$ & $\mathbf{3 4 . 2 3 6}$ \\
\hline \multicolumn{1}{|c|}{ Agrícolas } & 1,9 & 2,4 & 1,6 & 2,2 & 2,4 & 2,7 & 3,6 & 2,4 & 1,8 & 2,1 & 2,1 \\
\hline TRADICIONALES & 74.6 & 77.4 & 77,1 & 75,0 & 76,5 & 77.8 & 77.5 & 75.1 & 73,6 & 70,0 & 68,0 \\
\hline Agropecuarios & 5,8 & 5,1 & 5,4 & 6,2 & 6,8 & 6,2 & 6,1 & 6,7 & 8,0 & 10.7 & 12,8 \\
\hline $\begin{array}{l}\text { NO } \\
\text { TRADICIONALES }\end{array}$ & 24.6 & 22.2 & 22.5 & 24.4 & 22.9 & 21.5 & 21.9 & 24.2 & 25,8 & 29,5 & 31,7 \\
\hline OTROS & 0.8 & 0.4 & 0.4 & 0.6 & 0.6 & 0.7 & 0.7 & 0.7 & 0,6 & 0,5 & 0,3 \\
\hline TOTAL & 100.0 & 100.0 & 100.0 & 100.0 & 100.0 & 100.0 & 100.0 & 100.0 & 100,0 & 100,0 & 100,0 \\
\hline
\end{tabular}

Fuente: Banco Central de Reserva del Perú. Memoria Institucional 2015

La presencia de la inversión privada en la agricultura origina una modificación en la matriz agraria peruana, la cual en los últimos cinco 
años se ve impulsada por nuevos productos para el consumo extranjero, abordando nuevos mercados. Al principal producto agrícola peruano, el espárrago, se suma la uva que en el 2015 pasó a ser el mayor aportante de divisas en la categoría de productos agrícolas no tradicionales (Cuadro 3). Tal como expresa Porter (2013) el inversionista considera en esta diversificación los beneficios y los costos que implica participar en estos nuevos mercados. Es esta la circunstancia que debe considerar el gobierno central como resultado de conocer detalladamente los requerimientos de los consumidores internacionales.

\section{Cuadro 3}

Principales productos de agroexportación no tradicionales (Millones de US\$)

\begin{tabular}{|l|r|r|r|r|r|r|r|r|r|r|r|}
\hline & 2005 & 2006 & 2007 & 2008 & 2009 & 2010 & 2011 & 2012 & 2013 & 2014 & 2015 \\
\hline Quinua & n. d. & & 2 & 5 & 7 & 13 & 24 & 30 & 79 & 196 & 143 \\
\hline Espárragos frescos & n. d. & 187 & 236 & 228 & 251 & 291 & 292 & 340 & 412 & 385 & 416 \\
\hline Uvas & n. d. & 51 & 60 & 83 & 136 & 180 & 301 & 354 & 450 & 643 & 690 \\
\hline Paltas & n. d. & & 47 & 73 & 64 & 85 & 164 & 137 & 185 & 304 & 304 \\
\hline Mangos & n. d. & & 63 & 63 & 71 & 89 & 115 & 118 & 133 & 138 & 194 \\
\hline Bananos orgánicos & n.d. & & 31 & 46 & 51 & 49 & 64 & 81 & 89 & 119 & 145 \\
\hline
\end{tabular}

Fuente: Banco Central de Reserva del Perú - Memorias institucionales

Aparte del espárrago, tomando en cuenta el monto exportado en dólares americanos en el 2007, es sorprendente la cifra que obtiene en el período 2011 -2015. Además se tiene uvas de mesa, paltas, mangos y bananos orgánicos. El Banco Central de Reserva del Perú (2015, p. 60) informa que en "los últimos diez años, las ventas de productos agropecuarios han crecido a una tasa promedio anual de 15,8 por ciento, con lo cual, el valor exportado de 2015 fue aproximadamente más de tres veces el de 2006". Es esta evolución lo que ha permitido la modificación de la matriz exportadora de productos agrícolas. Por ejemplo, en el Cuadro 3 se observa el crecimiento de las ventas de la quinua al mercado internacional, el cual es un producto innovador en el mercado internacional y con aceptación por los consumidores en el exterior.

Monge (2012, p. 272) expone acerca de este camino hacia la "diversificación con valor agregado de la matriz productiva" que la realidad indica que debe realizarse una triple transición, de sectores, de actores y 
de mercados. En cuanto a sectores, que la economía peruana no dependa de la extracción y exportación de productos primarios, sino que apueste por la agricultura, la ganadería, la pesca, entre otros. Respecto a los actores que apuesten por la agricultura y la pesca, que tenga mayor participación los medianos y pequeños productores, así como interrelacionar a los productores de la costa, sierra y selva; no todo son los grandes grupos empresariales. Y en el caso, de la transición de mercados, dar prioridad a las necesidades locales.

Encaminar la producción del país a una etapa siguiente, tal como expone Monge (2012) es un cambio relativo de la matriz productiva que depende de las decisiones políticas del Estado, el nivel de riesgo aceptado por los inversionistas y la adaptabilidad del mediano y pequeño empresario para inmiscuirse en el proceso. La estrategia que se plantea el PNDP, implica producir más para vender más en el mercado internacional, en tipo de productos, en volumen (expresado en TM) y en montos (en US\$), de productos distintos a los minerales, que son importantes para la economía peruana, pero el grado de dependencia implica un riesgo que debe ser disminuido.

Sin embargo, es importante considerar la observación que realiza CEPAL (2016, p. 96) en cuanto a que Latinoamérica "tiene una débil participación en las cadenas globales de valor. Por un lado, su peso como origen de valor agregado extranjero contenido en las exportaciones mundiales (encadenamientos hacia adelante) continúa siendo muy pequeño". La observación lleva a que los países, lo que incluye Perú, reflexionen a pasar a un estadio en el cual los productos no tradicionales comiencen a tener un mayor valor agregado, lo cual motiva realizar -por parte del Estado- investigaciones en el mercado internacional acerca de que es lo que necesita el mercado y si mejora la rentabilidad del inversionista.

La participación de los productores locales en cadenas de valor, tal como expresan Fort y Vargas (2015) es un cálculo de beneficios y costos, cualitativos y cuantitativos, lo cual origina un proceso complicado de toma de decisiones. La duda es mayor en los pequeños agricultores, de ahí la importancia de la asociatividad, como un proceso de disminución de costos. Los gobiernos de estos años iniciales del siglo XXI han establecido lineamientos de política al respecto en la agricultura que se relacionan al 
cambio de la matriz de producción, considerando que esta acción permite generar economías de escala y tener una mejor capacidad de negociación.

La matriz agrícola es modificada por las oportunidades que hay en el mercado, siempre y cuando existan las condiciones locales que lo permitan. Es un proceso en el que hay que considerar aparte de los factores controlables aquellas ocurrencias imprevistas que son posibles de aprovechar. En tal sentido, el Estado debe promover, como parte de su política sectorial, la formación de una cadena de valor en que todos los participantes obtengan un beneficio. Además, debe promover mecanismos que permitan integrar a nuevos agentes productivos, en especial los pequeños agricultores, pudiendo ser aplicada una de las diversas modalidades de asociatividad.

\section{El pequeño agricultor en la competencia internacional}

La importancia del pequeña agricultura en los negocios internacionales, la expone el Ministerio de agricultura y riego - MINAGRI (2016) en el documento Política Nacional Agraria (PNA), en donde se expone la importancia de integrar a los pequeños agricultores, en el nuevo proceso comercial, porque son ellos el inicio de la cadena de la seguridad alimentaria de nuestro país, lo que muestra su posición estratégica. $\mathrm{Su}$ reconocimiento tiene consecuencias en los aspectos sociales y económicos de los agricultores e implica la preocupación por la mejora de sus condiciones de vida.

Las ideas de política de Estado están expuestas en el PNA, siendo importante citar que los lineamientos que se exponen están asociados a los pequeños agricultores, lo que puede tener el doble significado de lo complicado es que los pequeños agricultores se integren a la comercialización internacional o el Estado lo asume como un sector en el cual ahí sí puede influir de manera decidida y darle una orientación competitiva. La política de Estado es de influencia directa hacia un grupo de productores que se identifica que puede tener complicaciones para ser parte de la cadena exportadora.

Es evidente que la agricultura peruana está modificando y modernizando su matriz productiva como consecuencia de tener conocimiento y aceptar las tendencias de los compradores actuales y 
potenciales del mercado internacional. La consecuencia es obtener mayor provecho y que origine preocupación por este sea compartido, Ghezzi y Gallardo, (2013, p. 182) proponen que es necesario desarrollar el sector rural, poniendo énfasis en las zonas de sierra y selva, donde está el potencial de la nueva producción. Para los autores citados este es uno de los mayores desafíos del país. Son territorios que cuentan con abundante recurso hídrico el cual es limitado en la zona costera. Sin embargo, se debe administrar convenientemente de manera conjunta con los suelos por el efecto que pueda tener en el ambiente.

Es claro que para tal fin, aprovechar los territorios de la sierra y la selva, es necesario la creación de infraestructura (carreteras, electricidad, telecomunicaciones) que permita a estas regiones conectarse con las demás regiones de la costa. Esto es lo que permite incrementar las actividades emprendedoras y de esta manera los pobladores mejoran su bienestar e ingresos, así como se incrementa la oferta de los productos. El MINAGRI (2016, p. 24) que "un segmento importante de la agricultura peruana son los pequeños agricultores, con extensiones menores de 5 hectáreas, lo que equivale aproximadamente al 80 \% de los productores del país". El éxito de la agricultura peruana está relacionado a la integración de los diversos segmentos que forman parte de ella, iniciando por el pequeño agricultor.

El proceso por el cual se está realizando está en la etapa inicial de la reconversión productiva dentro del sector, en el cual se identifica dos actividades productivas. Una de ellas a cargo de los pequeños agricultores que se relaciona a la producción de los productos de consumo interno relacionados al aseguramiento alimentario. La otra actividad se relaciona a la producción para los mercados en el exterior. Tal como se propone en el documento Política Nacional Agraria del MINAGRI (2016) debe existir la posibilidad de que estos dos grupos productores se integren. Mientras más integrados estén los agentes del sector mayor y mejor será la cantidad de familias beneficiadas.

La tarea es compleja, en el sentido que se tienen que integrar una diversidad de conductas y acciones que son particulares en cada uno de los agentes de modo tal que se logre el objetivo que se plantea en relación a competir internacionalmente. Implementar la estrategia en la que los pequeños, medianos y grandes productores agrarios, el estado y los otros 
agentes que son parte de la cadena de valor tienen diferente forma de apreciar su aplicación es un desafío que debe asumirse. Thompson y otros (2015) proponen diez tareas básicas para llevar a cabo la estrategia sea cualquiera las condiciones del contexto, que se identifican en la Figura 3, las que están sujetas a la presión de los grupos de interés (stakeholders). En parte, coincide con la propuesta de Alarco (2012) en cuanto a contar con una organización que integre el esfuerzo de los participantes.

Optar por una estrategia de comercio internacional en la que el país tiene una cartera de productos diversificada, que incluye a los productos mineros, implica contar con personas que lleven a que las conductas de los participantes se oriente a obtener resultados exitosos en el comercio internacional, actividad en la que la competencia es intensa. El estilo con que los profesionales lleven a cabo las tareas expuestas tendrá un impacto directo en los resultados. Sus decisiones están entre la línea de dos puntos opuestos: el éxito y el fracaso. Los logros son esperados por los diversos grupos de interés quienes deben compatibilizar a la espera que el resultado favorezca a todos.

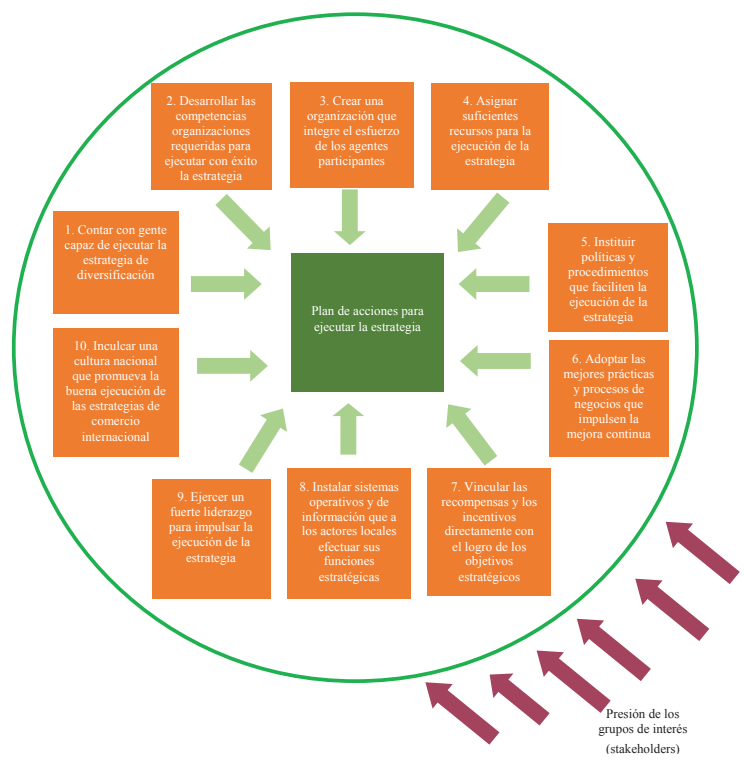

Figura 3. Tareas básicas para la ejecución de la estrategia Elaboración: propia en función a la propuesta de Thompson y otros (2015, p.262) 
El proceso es complejo porque implica un cambio estructural, es una nueva forma de pensar hacer cómo realizar la diversificación y tal como cita Machado (2014), quien basándose en investigaciones acerca del proceso de diversificación, expone que Perú no posee un sistema de planificación que le permitan al país saber a dónde va en el largo plazo. En tal sentido, considera que el Centro Nacional de Planificación Estratégica (CEPLAN) "carece de capacidad técnica y financiera, y del músculo político necesarios para convertirse en una guía de largo plazo para el conjunto de políticas sectoriales". La dimensión del desafío, en cuanto a la diversificación productiva, implica en el actual accionar el riesgo de avanzar de una forma lenta, de forma dispersa o no tener acciones planificadas y que cada agente actúe de acuerdo a sus objetivos particulares.

\section{Desafíos de la agricultura en la competencia internacional}

Considerar que la agricultura peruana tiene su importancia estratégica debido a su capacidad de penetración en los mercados internacionales, está dejando de lado el importante papel de los pequeños agricultores quienes asumen el papel estratégico de ser la base de la cadena alimentaria local y que representan el 40,0 \% del PBI del sector agrícola. Es la fuerza productiva que asegura el abastecimiento de alimentos a la población de las zonas urbanas del país. Sin embargo, también es parte de los competencia internacional, es parte de la estrategia de marketing internacional del país.

La diversificación implica tomar en cuenta que en la venta de productos agrícolas la competencia es intensa y se deben aprovechar las oportunidades que conceden los otros proveedores. Por ejemplo, en el mercado del aguacate la competencia es por el mercado norteamericano; México tiene la ventaja de la distancia, Perú su producción en contraestación, y en este momento aparece otros proveedores con quien con competir como Sudáfrica, Argentina y Chile, tal como informa MINAGRI (2015). Este comportamiento de complementariedad implica la exigencia a los productores locales de que su producción esté en condiciones de ser exportada en el momento que se requiere.

La pequeña agricultura tiene problemas estructurales que deben ser superados para que su integración se convierta en una realidad y así estén 
capacidad también de obtener los beneficios potenciales del comercio internacional. Las soluciones son diversas en las actividades que están presentes en el aparato productivo, en la posibilidad de fortalecer y agrandar la frontera agraria. Un punto inicial en el proceso de integración puede estar en modificar la forma de actuar en cuanto a cómo gestionar la actividad agraria a ese nivel. Lo que propone Artega (2016, p. 39) es un tema que debe ser considerado para fortalecer el proceso y tomando en cuenta que la diversificación implica un cambio estructural en el proceso de producción:

Es necesario transitar del enfoque predominante de ver la agricultura centrada en la fase productiva a un enfoque en el que la agricultura es una actividad económica como cualquier otra. Ello implicaría cambiar la denominación de productor por el de empresario agrario, sea agrícola, ganadero o forestal, con extensión a la fase de transformación. Se trata de una relación bidireccional; es decir, verlo como empresario y que él mismo se sienta emprendedor.

En el diagnóstico que realiza Arteaga (2016, p 40) identifica cuatro tipos de empresarialismo agrícola (Cuadro 4), cada uno con un sistema de producción que lo identifica, cartera de productos y diferentes mercados que atender. Lo que denominan "Unidades empresariales Consolidadas" son las que tienen su producción orientada al mercado internacional con productos que se han adaptado y adoptado de acuerdo a los consumidores en el exterior; podemos considerarlos como los innovadores de la industria, lo que incluye la aplicación de los avances tecnológicos. Las "Unidades empresariales parcialmente desarrolladas" tienen una cartera que mezcla productos locales con otros no nativos, y respecto al producto bruto interno del sector agrícola son los que realizan el mayor aporte; incluye a productores de productos agrícolas tradicionales como el café y la caña de azúcar, pero con una reorientación de mercado objetivo.

El tercer grupo "Unidades empresariales en formación" son productores en volumen para el mercado local, representan el 36,0 \% del PBI agrario. El cuarto grupo son las "Unidades desarticuladas del mercado" cuyo aporte al PBI agrícola es 4, 0 \% y se relaciona al autoconsumo. Son estos dos últimos la preocupación del Estado en cuanto a cómo articularlos a los citados en el párrafo anterior. Ambos representan el40 \% del PBI del sector agrícola y su producción en términos de costos unitarios es 
relativamente alta respecto a las unidades empresariales consolidadas y las que están en proceso.

\section{Cuadro 4}

Características, productos e importancia por tipo de agricultura empresarial

\begin{tabular}{|c|c|c|c|}
\hline $\begin{array}{l}\text { Tipo de } \\
\text { agricultura }\end{array}$ & Características predominantes & Cultivos & $\begin{array}{c}\text { Importancia } \\
\text { con respecto al } \\
\text { PBI del sector } \\
\text { agrícola } \\
\end{array}$ \\
\hline $\begin{array}{l}\text { Unidades } \\
\text { empresariales } \\
\text { consolidadas }\end{array}$ & $\begin{array}{l}\text { Producción intensiva con tecnología moderna. } \\
\text { Inserción en cadenas de valor. } \\
\text { Plenamente articulado al mercado externo e } \\
\text { interno, dinámico. } \\
\text { Certificaciones logradas para varios tipos de } \\
\text { mercado. } \\
\text { Alto nivel de gestión institucional. } \\
\text { Gran facilidad para financiamiento de la banca } \\
\text { de multiservicios } \\
\text { Rentabilidad significativa } \\
\text { Sostenibilidad del negocio. }\end{array}$ & $\begin{array}{l}\text { Mango, páprika, } \\
\text { olivo, espárrago, } \\
\text { alcachofa, banano } \\
\text { orgánico, uva. }\end{array}$ & $\begin{array}{l}10 \% \text { PBI } \\
140,000 \text { ha } \\
(5.5 \%)\end{array}$ \\
\hline $\begin{array}{l}\text { Unidades } \\
\text { empresariales } \\
\text { parcialmente } \\
\text { desarrolladas }\end{array}$ & $\begin{array}{l}\text { Producción extensiva. } \\
\text { Inserción en cadenas de valor. } \\
\text { Articulados a mercados internos dinámicos. } \\
\text { Certificaciones mínimas para ser abastecer a las } \\
\text { exportadoras. } \\
\text { Hábiles para el financiamiento formal e informal. } \\
\text { Gestión empresarial semidesarollada } \\
\text { Rentabilidad por volumen. }\end{array}$ & $\begin{array}{l}\text { Papa, arroz, maíz } \\
\text { amarillo duro, café, } \\
\text { caña de azúcar, } \\
\text { algodón. }\end{array}$ & $\begin{array}{l}50 \% \text { PBI } \\
1 ' 200,000 \text { ha } \\
(47.2 \%)\end{array}$ \\
\hline $\begin{array}{l}\text { Unidades } \\
\text { empresariales } \\
\text { en formación }\end{array}$ & $\begin{array}{l}\text { Producción a pequeña y mediana escala. } \\
\text { Articulados a mercados de consumo directo. } \\
\text { Canalizan financiamiento de banca de fomento. } \\
\text { Gestión empresarial incipiente. } \\
\text { Rentabilidad muy impactada por el mercado. }\end{array}$ & $\begin{array}{l}\text { Cebolla, tomate, } \\
\text { plátano, yuca, maíz } \\
\text { choclo, alfalfa. }\end{array}$ & $\begin{array}{l}36 \% \text { PBI } \\
800,000 \text { ha } \\
(31.5 \%)\end{array}$ \\
\hline $\begin{array}{l}\text { Unidades } \\
\text { desarticuladas } \\
\text { del mercado }\end{array}$ & $\begin{array}{l}\text { Producción muy fragmentada. } \\
\text { Producción con baja tecnología. } \\
\text { Sin acceso a servicios del Estado. } \\
\text { Dificultad para el financiamiento. } \\
\text { Orientada al autoconsumo e intercambio. } \\
\text { Descapitalización continua. }\end{array}$ & $\begin{array}{l}\text { Trigo, cebada, oca, } \\
\text { cebada en grano, } \\
\text { haba en grano. }\end{array}$ & $\begin{array}{l}4 \% \text { PBI } \\
400,000 \text { ha } \\
(15.7 \%)\end{array}$ \\
\hline
\end{tabular}

Fuente y elaboración: Arteaga (2016, p. 40)

La política de diversificación productiva puede considerar la propuesta de Arteaga, pero el proceso para su consolidación tiene que tener en cuenta: 1) compromiso de los productores y el Estado de conocer a todos los integrantes del proceso; 2) superar los problemas estructurales de los pequeños agricultores; 3) los principales agentes del 
aparato productivo deben estar articulados para compartir los beneficios; 4) estar seguro que la matriz productiva debe ser modificada de acuerdo a un plan, y 5) investigar para conocer la posibilidad de ingresar al mercado internacional de productos con mayor valor agregado. También debe considerarse la propuesta de Ghezzi (2016, p. 20) cuando expresa que "la idea no es diversificar por diversificar: Uno debería añadir activos a un portafolio solamente en la medida en que aumenten la rentabilidad esperada (ajustada por volatilidad o riesgo) del portafolio".

Al diversificar la cartera de productos agrícolas se adapta a las necesidades del comercio internacional y a las oportunidades que se presentan y por tanto el país debe estar en capacidad de aprovecharlas. Salvador (2016) menciona que "la globalización y los cambios económicos se manifiestan en un aumento de la población urbana y un incremento en la demanda alimentos procesados por cambio de hábitos de consumo". Aprovechar la oportunidad de los cambios en la economía internacional implica el fortalecimiento de la modificación de cambios relativos en la matriz productiva. A no ser que los agentes económicos decidan mantener la situación de productos no manufacturados. Por tanto, es necesario investigar para conocer la ventaja, la posibilidad y capacidad que se tiene en el nuevo ambiente. El fortalecimiento de la industria agrícola está en transitar, en un el mediano plazo a una posición relacionada con la manufactura.

Ghezzi (2016, pp. 20,21) recomienda que la diversificación de la producción tiene que estar acompañada por una diversificación de la cartera de compradores, pues al ser pocos cualquier ocurrencia en su comportamiento económico afecta la economía peruana. La diversificación es una forma de reducir la volatilidad de la economía peruana. Otro beneficio de la diversificación es que al ser generadora de nuevos sectores origina empleo formal y de calidad, lo que trae como consecuencia crecimiento en la producción y en el empleo formal. Añade que otro beneficio es que permite un desarrollo más balanceado en regiones mineras, hidrocarburiferas o gasíferas. "Pero además ofrece una oportunidad aún mayor para regiones que carecen de estas materias primas". 
El desafío principal para consolidar la diversificación está en la identificación de los productores, productos y momentos en que aparecen los requerimientos de los compradores internacionales y si Perú está en capacidad de realizarlo, de modo que las oportunidades otorguen resultados favorables al país. A la par, debe ir la educación del recurso humano en cuanto el conocimiento permite romper barreras culturales, incentivar el incremento de la productividad, propicia la posibilidad de mejorar el bienestar de las familias y por tanto, aumento de la demanda local con exigencias más y mejores bienes y servicios. El proceso exige que se tomen las medidas necesarias que ayuden a consolidar los pasos propuestos en donde el sector empresarial es la clave.

\section{Apreciaciones finales}

La competencia internacional a nivel país implica un cambio en el sistema de producción. Es diferente competir en un mercado de commodities que en un mercado de productos innovadores, que siguen siendo primarios en la medida que no se realiza -en la mayoría de ellos transformación alguna. Para lograr resultados sostenibles tiene que entrarse en un proceso de diversificación de la producción en donde se tome en cuenta los distintos actores de la cadena de valor de cada uno de los productos. Es decir, de alguna manera tiene que realizarse una acción que centralice los esfuerzos y necesidades de los actores.

La diversificación es una característica del sistema de producción de un país. Lo que interesa es la intensidad y compromiso de los inversionistas innovadores asumen un riesgo por la novedad, así como el soporte de las organizaciones del Estado. A nivel macro, lo que debe considerarse como diversificación es qué tanto valor relativo tiene la innovación dentro de la matriz de producción. Es importante el cambio relativo, que implique un aumento de producción en los commodities y en los productos no tradicionales. Perú tiene posibilidades de ese tipo de cambio en el sector agrario. Si así fuera hay decisiones que deben realizarse en cuanto a la interrelación de todos los agentes de la cadena de valor, lo que incluye al Estado y a los pequeños agricultores.

La competencia internacional entre países implica que tan fuerte son las cadenas de valor al interior del país. El pequeño agricultor es un eslabón de la cadena local que cumple un papel importante pues es 
el que asegura el aprovisionamiento del mercado interno, facilitando así el accionar del Estado para que promueva las exportaciones. La acción es de aseguramiento del abastecimiento interno para lo cual lleva a cabo actividades de desarrollo que le permiten mejorar su producción. También, establece un marco que permite a los inversionistas y productores en mediana y gran escala proveer los mercados extranjeros.

Los desafíos de la competencia internacional, son amplios. Porter (2009, p. 227) expone que "las empresas logran ventaja competitiva mediante actos de innovación. Enfocan la innovación en su sentido más amplio, que comprende tanto nuevas tecnologías como nuevos modos de hacer las cosas". La diversificación productiva, como parte de una estrategia país, es un camino de competencia intensa que permite demostrar ese espíritu innovador. La potencialidad de producción en la agricultura es amplia, en cuanto hay para producir más de lo mismo y aquello que todavía no se realiza. Lo mismo ocurre ,en cuanto a potencialidad, con los mercados, los cuáles buscarán a su mejor proveedor, en cuanto a marca país, cantidad y calidad del producto, estación o momento, precios, entre otros.

\section{Referencias bibliográficas}

Alarco, G. (2015). Tecnopolítica económica: análisis y propuestas. Lima: Universidad del Pacífico.

Alarco, G. (2012). Diversificación productiva en el Perú: retos y alternativas. Libro: Caminos de transición, alternativas al extractivismo y propuestas para otros desarrollo en el Perú, pp. 219 - 262. Lima: Red Peruana por una globalización con equidad (RedGE). Disponible en: http://www.redge.org. pe/sites/default/files/Caminos\%20de\%20Transici\%C3\%B3n\%20-\%20 RedGE_PDF_low.pdf

Arteaga, W. (2016). Gestión del sector agricultura al 2021: hacia un desarrollo sostenible. Libro: Metas del Perú al Bicentenario. Lima: Consorcio de universidades. Url: http://www.consorcio.edu.pe/metas-bicentenario/ Metas-del-Peru-al-Bicentenario-Consorcio-de-Universidades-Libro-Digital. pdf

Banco Central de Reserva del Perú (2015). Memoria Institucional. Lima: Banco Central de Reserva del Perú. Disponible en: http://www.bcrp.gob.pe/docs/ Publicaciones/Memoria/2015/memoria-bcrp-2015-2.pdf 
Barrientos, P. (2014). El desarrollo de la marca país: base para posicionarse a través de las exportaciones no tradicionales. Bogotá: Finanzas y Política Económica, pp. 115 - 140.

Barrientos, P. (2016). Macromarketing en el Perú: la estrategia de diversificación productiva. Bogotá: Revista Equidad y Desarrollo, Universidad La Salle. Disponible en: https://revistas.lasalle.edu.co/index.php/ed/article/ view/3761

Comisión económica para América latina - CEPAL- (2016). Panorama de la inserción internacional de América Latina y el Caribe, la región frente a las tensiones de la globalización. Santiago de Chile: CEPAL.

Fort, R.; Vargas, R. (2015). Estrategias de articulación de los productores agrarios en la costa peruana: ¿asociatividad, vinculación con empresas o ambas?, incluido en el libro Agricultura peruana: nuevas miradas desde el censo agropecuario. Lima: Grade.

Ghezzi, P. y Gallardo, J. (2013). Qué se puede hacer por el Perú, ideas para sostener el crecimiento económico en el largo plazo. Lima: Universidad del Pacífico; Universidad Católica del Perú.

Ghezzi, P. (2016). Logros y desafíos de la diversificación productiva en el Perú. Lima: Ministerio de la Producción. Disponible en: http://citeagroindustrial. com.pe/es/images/logrosdesafios.pdf

Gómez, R. (2016). Agricultura y servicios ecosistémicos. Libro: Metas del Perú al Bicentenario. Lima: Consorcio de universidades. Disponible en: http://www. consorcio.edu.pe/metas-bicentenario/Metas-del-Peru-al-BicentenarioConsorcio-de-Universidades-Libro-Digital.pdf

Hausmann, R. e Hidalgo, C. (2010). Country diversification, product ubiquity and economic divergence. Boston: Working papers, Center for International Development at Harvard University.

Hausmann, R.; Rodrik, D. (2002). Economic development as self discovery. Cambridge: National Boreau of Economic Research

Kotler, P. y Armstrong, G. (2013). Fundamentos de marketing. Decimoprimera edición. México D. F.: Pearson Educación.

Machado, R. (2014). Hacia la diversificación y sofisticación productiva en el Perú. Lima: OXFAM

Ministerio de Agricultura y Riego (2015). Lineamientos de política agraria. Lima: Ministerio de Agricultura y Riego. Disponible en: http://www.minagri.gob. pe/portal/download/pdf/p-agraria/lineamientos.pdf 
Ministerio de Agricultura y Riego (2015). La palta, producto estrella de exportación. Tendencias de la producción y el comercio de palta en el mercado internacional y nacional. Lima: Ministerio de Agricultura y Riego. Disponible en: file://C:/Users/Director/Downloads/informe-paltaperuana-300115\%20(1).pdf

Ministerio de Agricultura y Riego (2016). Decreto Supremo Nº 002-2016-Minagri. Política Nacional Agraria. Lima. Ministerio de agricultura y riego. Disponible en: http://www.minagri.gob.pe/portal/download/pdf/p-agraria/politicanacional-agraria.pdf

Ministerio de la Producción (2014). Plan Nacional de Diversificación Productiva. Lima: Ministerio de la Producción. p. 126

Monge, C. (2012). Caminos de Transición (para salir del extractivismo). Libro: Caminos de transición, alternativas al extractivismo y propuestas para otros desarrollo en el Perú, pp. 263 - 276. Lima: Red Peruana por una globalización con equidad (RedGE). Disponible en: http://www.redge.org. pe/sites/default/files/Caminos\%20de\%20Transici\%C3\%B3n\%20-\%20 RedGE_PDF_low.pdf

Porter, M. (2013). Ser competitivo. Sexta Edición. Madrid: Ediciones Deusto.

Salvador, G. (2016). Agregado de valor, compartiendo conceptos. Chubut: Revista Economía y Mercados, pp. 75 - 81. Disponible en: http://inta.gob.ar/sites/ default/files/agregado_de_valor._compartiendo_conceptos.pdf

Thompson, A.; Gamble; J.; Peteraf, M.; Strickland, A. (2015). Administración estratégica, teoría y casos. Decimonovena edición. México D.F.: McGraw Hill/ Interamericana Editores S. A. de C. V. 